\title{
MBE GROWTH AND PROPERTIES OF GaMnAs(100) FILMS
}

\author{
J. Sadowski, J. Domagala, J..Bąk-Misiuk, K. Świątek \\ Institute of Physics, Polish Academy of Sciences \\ Al. Lotników 32/46, 02-668 Warsaw, Poland \\ J. KANSKI, L. ILver and H. Oscarsson \\ Department of Physics, Chalmers University of Technology and Göteborg University \\ 41296 Göteborg, Sweden
}

We present here the results of measurements of structural and electronic properties of GaMnAs - a new diluted magnetic semiconductor system. This ternary III-V-Mn compound with the Mn content as high as $7 \%$ was obtained for the first time (by means of molecular beam epitaxial growth) by Ohno, Munekata et al. and the studies of its properties are not completed until now. We did the high resolution X-ray diffraction investigations and photoemission measurements of the samples with $\mathrm{Mn}$ content varied from about $0.1 \%$ up to $5 \%$. The crystalline perfection of the ternary GaMnAs compound is very high - full width at half maximum of GaMnAs (400) Bragg reflections are of order of 50 arcseconds and the layers are fully strained to the $\mathrm{GaAs}(100)$ substrate. In photoemission experiments we traced the contribution of Mn $3 d$ states to the band structure of GaMnAs ternary compound.

PACS numbers: $81.15 . \mathrm{Hi}, 79.60 . \mathrm{Bm}, 71.55 . \mathrm{Eq}$

III-V diluted magnetic semiconductors (DMS), studied recently by Munekata, Ohno et al. $[1,2]$ have attracted much attention because of occurring of transition to ferromagnetic phase below temperature of $110 \mathrm{~K}$ - much higher than in the case of II-VI and IV-VI semiconductors containing $\mathrm{Mn}$.

In this work we present results of studies of GaMnAs layers with Mn content of $0.1 \%$ to $5 \%$. For MBE growth experiments a DCA MBE system located at the MAX-Lab synchrotron radiation facility in Lund, Sweden was used. The samples were grown by adding Mn flux during low temperature (at the substrate temperature of $250^{\circ} \mathrm{C}$ ) growth of GaAs. A DCA cracking cell was applied as an arsenic source. This cell generates $\mathrm{As}_{2}$ molecules and allows to use lower As pressures (about $(2-5) \times 10^{-9} \mathrm{Tr}$ ) in the MBE system. It is important because after MBE growth the samples are transported into the analytical chamber where the photoemission experiments are performed. Before the MBE growth the As, Ga, and 
Mn fluxes were calibrated by the ion gauge placed in the position of the substrate, and by reflection high energy electron diffraction (RHEED) intensity oscillations recorded during GaAs and GaMnAs growth. Semi-insulating GaAs(100) platelets were used as substrates. The growth of all samples was started with the high temperature (at a substrate temperature of about $600^{\circ} \mathrm{C}$ ) GaAs $0.2 \mu \mathrm{m}$ thick buffer. During growth of this buffer layer 2D RHEED pictures with $2 \times 4$ surface reconstruction and intense specular spot were observed. It is well known that the low temperature (LT) MBE growth of GaAs (at growth temperatures below $300^{\circ} \mathrm{C}$ ) should be done at high As/Ga flux ratio. To assess and adjust this ratio, the specular spot intensity oscillations were detected for different $\mathrm{Ga}$ fluxes. The Ga cell temperature used during LT GaAs as well as GaMnAs growth was chosen at a level corresponding to the $\mathrm{As}_{2} / \mathrm{Ga}$ flux ratio of about 12. Specular spot RHEED intensity oscillations recorded at 2 different temperatures of $\mathrm{Ga}$ cell during high temperature (HT) and LT GaAs buffer growth are shown in Fig. 1. The thickness

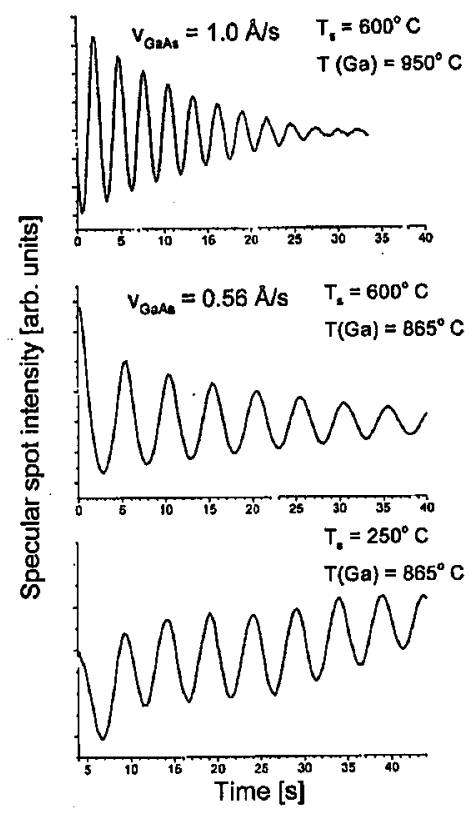

Fig. 1. RHEED intensity oscillations recorded during MBE growth of high temperature $\left(600^{\circ} \mathrm{C}\right)$ and low temperature $\left(250^{\circ} \mathrm{C}\right) \mathrm{GaAs}$ buffer layers.

of LT GaAs buffer was about $0.05 \mu \mathrm{m}$. After GaMnAs growth start a $(2 \times 1)$ surface reconstruction appeared and in the case of the samples with low Mn content some traces of $c(4 \times 4)$ reconstruction were also visible. The $\mathrm{Mn}$ temperature was changed from 680 to $780^{\circ} \mathrm{C}$. At $T_{\mathrm{Mn}}=790^{\circ} \mathrm{C}$ and $T_{\mathrm{Ga}}=920^{\circ} \mathrm{C}$, which corresponds to $\mathrm{Mn} / \mathrm{Ga}$ flux ratio of about 0.1 , a $\mathrm{Mn}$ segregation was observed, as the second monocrystalline phase seen in the RHEED picture. According to the literature data there were MnAs microcrystallite precipitates. The interesting point in this 
case was that the first $8 \mathrm{GaMnAs}$ monolayers grew without any traces of such precipitates and 8 specular spot intensity oscillations were recorded. Afterwards, the RHEED intensity decreased gradually, no more oscillations were observed and additional Bragg spots appeared in the RHEED picture.
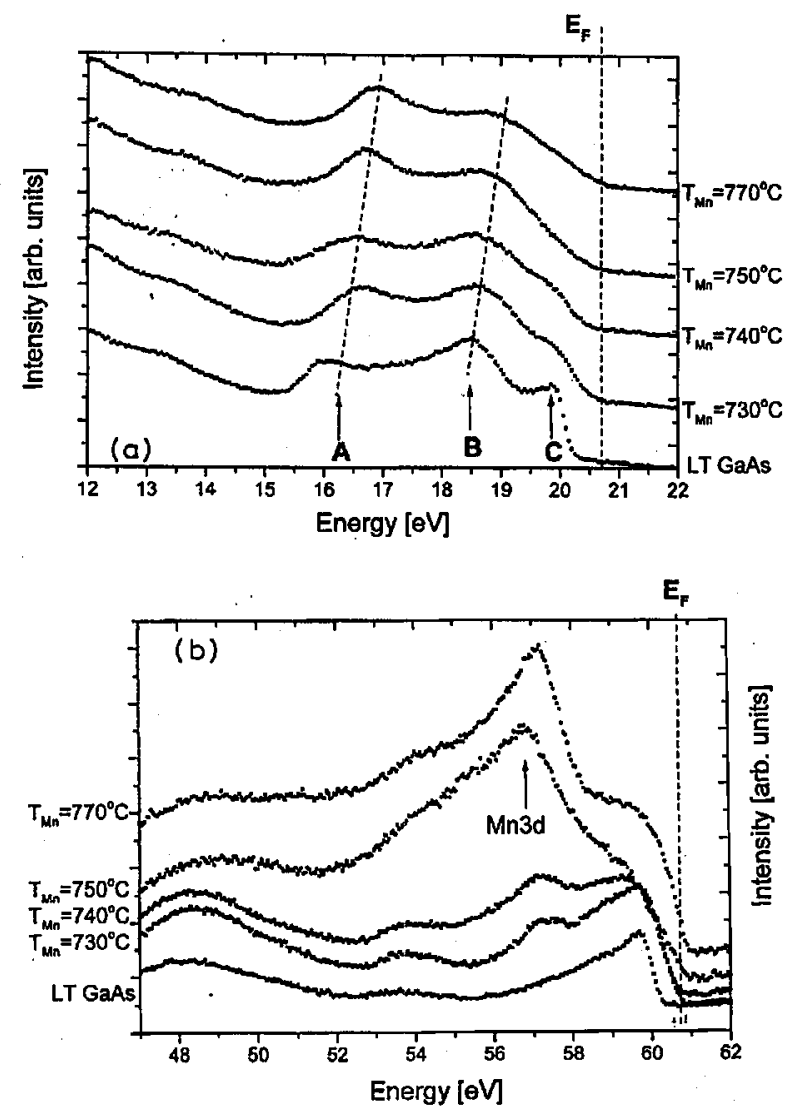

Fig. 2. Photoemission spectra for GaMnAs samples grown at different Mn effusion cell temperatures. (a) Excitation energy $25 \mathrm{eV}: A, B$ - maxima connected with GaAs bulk interband excitations, $C-c(4 \times 4)$ surface state, $E_{F}-$ Fermi energy, (b) excitation energy $65 \mathrm{eV}$.

After growth the samples with Mn content of $0.5 \%$ to $5 \%$ were transferred to the analytical chamber where the photoemission measurements were performed. A beam generated by $550 \mathrm{MeV}$ synchrotron was used as a light source. These measurements were done at 2 excitation energies: $25 \mathrm{eV}$ and $65 \mathrm{eV}$ (see Fig. 2a and $2 \mathrm{~b})$. In the spectrum corresponding to the excitation energy of $25 \mathrm{eV}$ three maxima denoted by $A, B$, and $C$ can be identified. Peaks $A$ and $B$ are connected with GaAs bulk interband excitations, peak $C$ with $c(4 \times 4)$ surface state. Consistently with the behaviour of the RHEED pictures, where this reconstruction was ob- 
served only for the samples with the lowest Mn content, this peak disappears with increasing $\mathrm{Mn}$ composition. Evolution of $A$ and $B$ peaks connected with GaAs bulk interband excitations reflects the modifications of the band structure of GaMnAs with increasing Mn content.

Two maxima visible in Fig. $2 \mathrm{~b}$ result from the resonant photoemission signal connected with Mn $3 d$ state. For samples with more Mn the high energy peak at about $60 \mathrm{eV}$ approaches the Fermi energy which indicates that the sample evolves in the "metallic" direction. That is consistent with the observation [3] of high hole concentrations (above $10^{18} / \mathrm{cm}^{3}$ ) in GaMnAs with $x_{\mathrm{Mn}}$ of order of several percent.
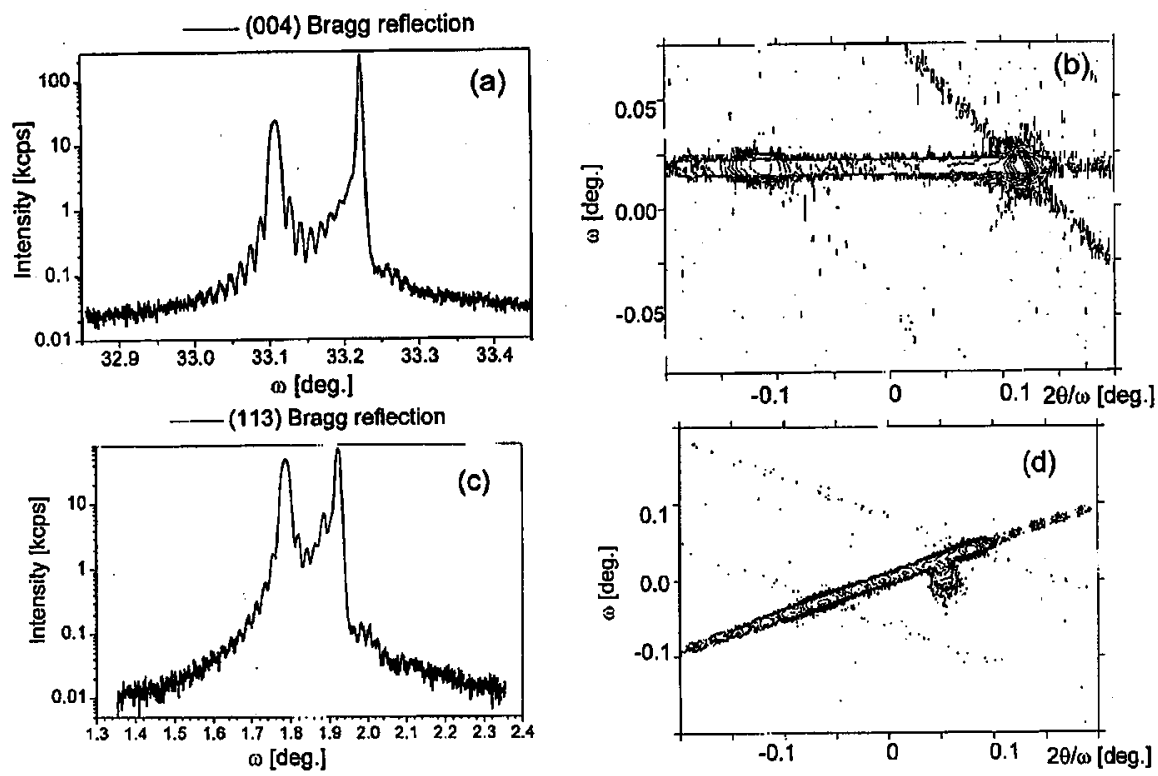

Fig. 3. High resolution X-ray diffraction measurements of the GaMnAs with $1 \% \mathrm{Mn}$ : (a) (400) $\omega / 2 \theta$ scan, (b) corresponding reciprocal space map, (c) (113) $\omega / 2 \theta$ scan, (d) corresponding reciprocal space map.

The results of X-ray diffraction measurements of sample with $1 \% \mathrm{Mn}$ are shown in Fig. 3. Figure 3a shows $\omega / 2 \theta$ scan of symmetric (400), Fig. 3c - asymmetric (113) Bragg reflection, Figs. $3 \mathrm{~b}$ and $3 \mathrm{~d}$ - corresponding reciprocal space maps. The full width at half maximum of (004) Bragg reflection is 56 arcseconds, which reflects the high structural perfection of the GaMnAs layer. Its parallel and perpendicular lattice constants are

$$
a_{\|}=5.653 \AA, \quad a_{\perp}=5.686 \AA .
$$

From reciprocal space maps it follows that the layers are fully strained to the substrate and there are no defects like mosaicity and no lattice relaxation in the GaMnAs sample studied (containing $1 \% \mathrm{Mn}$ ). 
The first transport measurements performed for the sample containing $2 \% \mathrm{Mn}$ gave the following results:

$$
\begin{aligned}
& T=300 \mathrm{~K} \text {, hole concentration } p=8 \times 10^{17} \text {, mobility } \mu=40 \mathrm{~cm}^{2} /(\mathrm{V} \mathrm{s}), \\
& T=77 \mathrm{~K} \text {, hole concentration } p=9 \times 10^{16}, \text { mobility } \mu=106 \mathrm{~cm}^{2} /(\mathrm{V} \mathrm{s}), \\
& T=4 \mathrm{~K} \text {, hole concentration } p=2 \times 10^{16} \text {, mobility } \mu=560 \mathrm{~cm}^{2} /(\mathrm{V} \mathrm{s}) .
\end{aligned}
$$

These results differ from the results of Ohno et al. $[2,3]$ by much lower carrier concentrations.

We have presented the first, to our best knowledge, results of in situ (i.e. without taking the samples out of the vacuum system) photoemission measurements of MBE grown GaMnAs layers with Mn content of $0.5 \%$ to $5 \%$. The evolution of Mn $3 d$ state (measured in the resonant photoemission mode) witl increasing $\mathrm{Mn}$ content has been presented. The high structural perfection of the grown GaMnAs samples has been confirmed by the results of high resolution X-ray diffraction experiments. The preliminary results of electrical parameters of GaMnAs layers have shown lower hole concentrations than reported so far in the literature.

The authors would like to thank Dr. W. Dobrowolski for the Hall measurements.

\section{References}

[1] H. Munekata, H. Ohno, S. von Molnar, A. Segmüller, L.L. Chang, L. Esaki, Phys. Rev. Lett. 63, 1849 (1989).

[2] F. Matsukura, H. Ohno, A. Shen, Y. Sugawara, Phys. Rev. B 57, R2037 (1998).

[3] A. Oiwa, S. Katsumoto, A. Endo, M. Hirasawa, Y. Iye, H. Ohno, F. Matsukura, A. Shen, Y. Sugawara, Solid State Commun. 103, 209 (1997). 